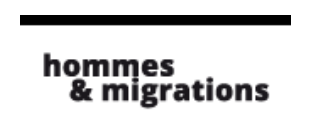

Hommes \& migrations

Revue française de référence sur les dynamiques

migratoires

$1290 \mid 2011$

Travailleurs sociaux et migrations

\title{
Ethnicité et sexuation ordinaire dans un foyer pour jeunes femmes en difficulté sociale
}

\author{
Wassim El Golli
}

\section{(2) OpenEdition \\ Journals}

\section{Édition électronique}

URL : http://journals.openedition.org/hommesmigrations/734

DOI : 10.4000/hommesmigrations.734

ISSN : 2262-3353

\section{Éditeur}

Musée national de l'histoire de l'immigration

\section{Édition imprimée}

Date de publication : 1 mars 2011

Pagination : $34-43$

ISSN : 1142-852X

\section{Référence électronique}

Wassim El Golli, «Ethnicité et sexuation ordinaire dans un foyer pour jeunes femmes en difficulté sociale », Hommes \& migrations [En ligne], 1290 | 2011, mis en ligne le 31 décembre 2013, consulté le 01 mai 2019. URL : http://journals.openedition.org/hommesmigrations/734; DOI : 10.4000/ hommesmigrations.734 


\section{Ethnicité et sexuation ordinaire dans un foyer pour jeunes femmes en difficulté sociale}

Par Wassim El Golli, doctorant, IRIS, EHESS, attaché temporaire à l'enseignement et la recherche, Lille-3

Chargés d'une autorité et d'une mission publiques, les travailleurs sociaux peuvent être conduits à relayer dans leur pratique certaines représentations négatives attachées aux populations dont ils ont la charge. Dans une maison éducative à caractère social, l'accompagnement de jeunes femmes d'origine étrangère n'échappe pas à une approche soumise au prisme du sexe et de l'origine ethnique.

La question du traitement de leur sexualité au sein du foyer permet de saisir le jeu des stéréotypes qui déterminent en sourdine les rapports sociaux. 
De nombreuses recherches en sociologie se sont intéressées au travail social et aux mécanismes de pouvoir à l'ceuvre dans le rapport au politique et dans la relation de proximité entre les acteurs. Dès les années soixante-dix, plusieurs auteurs ont porté le débat sur la mission et le sens de l'intervention de l'État dans le champ social ${ }^{(1)}$, et sur le rôle joué par les institutions et l'action sociale comme instruments "normalisateurs" sur des "populations déviantes ${ }^{(2) "}$. Dans les années quatre-vingt-dix, plusieurs travaux se sont intéressés à la profession des travailleurs sociaux ${ }^{(3)}$ ou au "mythe éducatif" en

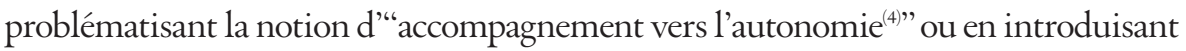
des notions comme la relation de service ${ }^{(5)}$ ou la désaffiliation ${ }^{(6)}$.

L'analyse du travail social sous l'angle des rapports de pouvoir, notamment ceux fondés sur la classe, a mis en évidence ses fonctions de contrôle social. Pour autant, ces travaux ont occulté longtemps d'autres rapports de domination comme le genre ${ }^{(7)}$ et la "race ${ }^{(8) "}$. Il faut en effet attendre les années deux mille pour voir des travaux questionnant les hiérarchies sexuées dans le travail social ${ }^{(9)}$ ou dans la relation d'aide et le travail sur autrui ${ }^{(10)}$. Cependant, malgré une importante production scientifique sur le sujet ces dernières années ${ }^{(11)}$, la question du genre dans le champ de l'analyse du travail social demeure marginale. Concernant la question des rapports sociaux de "race", le débat public comme académique demeure fermé(12), même si quelques chercheurs ont très tôt questionné l'impact des politiques publiques sur les migrant $(\mathrm{e}) \mathrm{s}^{(13)}$. Il faut attendre la deuxième moitié des années quatre-vingt pour voir apparaître des travaux sur le travail social et les migrants ${ }^{(14)}$. Encore plus récemment, des chercheuses ont croisé la question du genre avec celle de la "race" dans le cadre du travail sur autrui ${ }^{(15)}$.

Dans la continuité de ces travaux, nous tenterons de comprendre comment les questions de genre et de race agissent sur la relation de proximité et le travail avec autrui. À partir d'une enquête de terrain au sein d'une structure d'accueil d'adolescentes et de jeunes femmes (voir encadré), nous chercherons, dans un premier temps, à identifier la manière dont les catégories genre et "race" interviennent dans les rapports jeunes/institution. Nous verrons par la suite comment s'articulent genre et race dans le travail sur autrui à travers l'exemple de la sexualité.

\section{"L'alibi de la nature" et du sens commun : la sexuation au quotidien}

Parler de rapports sociaux de genre dans l'institution, c'est identifier les enjeux sexués, des processus sociaux à l'ceuvre entre les acteurs. Pour comprendre les enjeux de l'accompagnement éducatif, il faut avoir à l'esprit que les éducateurs ne sont pas 
seulement un groupe homogène mais aussi divisibles en deux classes ${ }^{(16)}$, hommes et femmes. Cette division tire ses origines d'une lecture structurelle de la division sexuelle du travail. La répartition des tâches domestiques entre les différents éducateurs en est un exemple classique. Même si aucun document ne spécifie les rôles à l'intérieur du foyer, les éducatrices ont souvent reproché, au cours des réunions d'équipe, à leurs collègues masculins de ne pas avoir fait le ménage. Françoise dit ainsi sur un ton agacé: "Je suis arrivée le lundi matin, jai trouvé le foyer dans un tel état! Je ne

La réflexion présentée dans cet article s'inspire d'une recherche en cours sur les pratiques éducatives pour une thèse de sociologie.

Dans ce cadre, j'ai été embauché comme élève éducateur en formation sur un poste d'éducateur de nuit pendant plus de deux ans dans une Maison éducative à caractère social (MECS) de la région parisienne. Cet établissement est un foyer spécialisé dans la prise en charge éducative d'adolescentes et de jeunes femmes de 15 à 21 ans placées par voie juridique (placement direct du juge pour enfants ou du juge de la famille) ou administrative (Aide sociale à l'enfance). Ce foyer accueille 30 jeunes femmes. L'apport d'une approche inductive et participative pour une telle enquête est double : la proximité avec les acteurs, surtout lorsqu'on touche à la situation personnelle des jeunes femmes, voire à leur intimité, et la participation à la pratique éducative, en étant présent au quotidien. L'ethnographie réalisée dans ce lieu a privilégié l'utilisation d'outils méthodologiques spécifiques, le journal de terrain, les entretiens et la consultation des documents internes à la structure. C'est pourquoi les noms des jeunes femmes et des travailleurs sociaux cités dans cet article ont été modifiés et les détails qui pourraient identifier le foyer ont été cachés. comprends pas, à chaque fois que Pierre fait le week-end c'est pareil, ce n'est pas à nous [les éducatrices] de nous occuper de ça et d'organiser des tours de ménage avec les filles.' La spécificité du travail dans le foyer est ce mélange entre un espace public professionnel et un espace privé intime lié à la nature du travail de proximité Ainsi la division sexuelle du travail à l'intérieur du foyer ressemble fortement à ce qu'on voit à l'extérieur.

Ces tensions entre les éducateurs dépassent la question des répartitions des tâches et se répercutent sur le travail d'accompagnement et les relations avec les jeunes. Lorsque Pierre n'organise pas les tours de ménage du week-end, les éducatrices sont doublement ramenées à leur position de femmes quant à l'exécution des tâches et à la manière dont les jeunes femmes les perçoivent. En effet, ces dernières ont bien compris les tensions entre les 
deux sexes dans l'équipe et ne se privent pas de rappeler aux éducatrices que les hommes ne font rien et que ce sont les femmes qui s'occupent du ménage. Cette division sexuelle du travail conforte donc les processus sociaux existants dans la société et les assignations de genre.

En puisant dans l'imaginaire commun, des choses connues de tous ou, comme le nomme Delphy, "l'alibi de la nature ${ }^{(17),}$, le discours des éducateurs reproduit les représentations et les stéréotypes sociaux qui renforcent un discours normatif et biologisant. Ainsi, courant 2008, une jeune fille du foyer a été agressée dans son lycée par un garçon de sa classe et, pendant la réunion suivant cet incident, les éducateurs ont questionné les tenues "osées" de Carla et son tempérament "hystérique lorsqu'elle perd le contrôle ${ }^{(18) "}$. Même si l'équipe n'a pas limité son analyse à ces deux aspects et a, au contraire, cherché à contextualiser la situation de Carla, le fait de mentionner ces raisons nous renseigne sur les représentations que se font les travailleurs sociaux des jeunes femmes du foyer. Ici aussi les éducateurs utilisent des analyses sexuées et une lecture normative du "sexe" en reprenant les stéréotypes ordinaires de catégorisation et de différenciation à l'encontre des femmes.

Cachée dans les plis d'un quotidien parfois sans enjeux, cette parole naturalisante et sexuée n'est pas toujours visible : il faut être conscient des rapports de pouvoir et de domination sous-jacents pour la démasquer. Cette invisibilité n'est pas seulement d'ordre individuel, elle est aussi une construction sociale ${ }^{(19)}$ et institutionnelle dans laquelle le foyer n'est qu'un aspect. Lorsque l'on regarde les orientations scolaires des jeunes filles au foyer, on remarque que plus des deux tiers sont inscrites dans des filières professionnelles à forte tendance féminine (coiffure, esthétique, carrière sanitaire et sociale ou vente). Comme l'ont montré plusieurs recherches, à l'école, à l'hôpital ou dans la sphère domestique, il y a un processus de différenciation fondé sur le sexe qui participe à une domination spécifique des femmes ${ }^{(20)}$ et le foyer n'échappe pas à ces rapports de pouvoir et de domination.

\section{L'ethnicité, un processus ordinaire}

Le foyer est un terrain privilégié pour étudier les normes implicites et leurs impositions $^{(21)}$. Parler d'ethnicité dans ce contexte n'est ni une tentative de masquer des pratiques racistes ou racisantes, ni un demi-mot scientifique pour qualifier une dérive institutionnelle, mais plutôt un outil pratique pour comprendre la relation entre dominant et dominé, dépositaire de l'action sociale et usager. Le dernier rapport d'activité fait état d'une "forte progression d'un public africain (14 jeunes sur 19 jeunes accueillies $)^{22}$ ". Ce traitement différencié renvoie à un ailleurs indéfini et homogénéisant 
où les nuances entre les différents parcours sont gommées pour laisser place à un imaginaire figé et stéréotypé. Anne Loyer affirme ainsi que "l'individu est généralement perçu comme un membre représentant de son groupe d'appartenance et donc à travers l'image et le stéréotype que nous avons de ce groupe $e^{(23) !}$.

Pourtant, cette invisibilisation de la "race" n'est pas toujours pertinente puisque la différence est parfois mise en scène. Ainsi, lors d'une sortie éducative avec les jeunes, il fallait, pour des raisons pratiques, organiser un déplacement en deux groupes, le tout dans une ambiance tendue en raison du froid ce jour-là. Alors qu'un premier groupe prend place dans le véhicule, un éducateur décide de faire descendre une des jeunes pour laisser la place à une autre, en précisant que "la peau noire supporte moins le froid que la blanche ${ }^{(24)}$. Avant de se justifier, “à cause de la pigmentation de la peau”. Dans ce cas, le professionnel met l'accent sur la nature et la science pour justifier un traitement différencié. Comme on l'a vu pour les différenciations sexuées plus haut, la nature sert aussi d'alibi pour justifier une catégorisation racialisée ${ }^{(25)}$.

Au foyer, la différenciation entre les jeunes femmes sur des bases ethniques peut être aussi une question institutionnelle, comme l'illustre l'attitude d'un éducateur un jour de Ramadan. Il a refusé de retarder le dîner d'un quart d'heure pour que les jeunes femmes qui jeûnent puissent manger avec le reste du groupe et les a obligées à prendre place autour de la table et à attendre la rupture du jeûne assises devant les plats. Cela au nom du règlement, du respect de l'heure du dîner et du caractère läque de l'institution. Sous le prétexte du maintien du cadre et de la défense d'une certaine norme institutionnelle, l'universalisme peut servir d'alibi pour stigmatiser un groupe à partir d'une différenciation ethnique qui se rapproche dans ce cas du racisme.

À travers ces quelques exemples, on voit comment des rapports sociaux ethnicisés peuvent exister à l'intérieur d'un foyer même si les documents officiels ne le montrent pas. Ainsi, on a pu voir qu'en invisibilisant des rapports sociaux, en les légitimant ou en distinguant les jeunes femmes selon leur statut administratif, la différenciation sur des bases raciales et ethniques est une réalité du travail de proximité.

\section{La sexualité des jeunes femmes entre maîtrise, prévention et contrôle}

L'institution intervient sur le parcours des jeunes selon des schémas sexués, à travers des injonctions au quotidien ou en questionnant les liens familiaux et les choix professionnels. De même, l'institution ne sort pas du cadre de pensée biaisé par des considérations de "race", comme celles évoquées ci-dessus. Ces enjeux de pouvoir peuvent aussi se conjuguer dans un même schéma, sans une prédominance de l'un 
sur l'autre mais plutôt avec une intersectionalité où la "race" et le genre interviennent simultanément dans les rapports sociaux ${ }^{(26)}$. Pour illustrer ces propos, nous nous proposons d'analyser le traitement de la sexualité dans le foyer afin de comprendre comment ces rapports de pouvoir sont inhérents au travail social en institution.

La sexualité est principalement abordée dans le foyer par le prisme de la prévention et de l'information. Ainsi, dans le compte rendu d'une intervention d'élèves infirmières sur les maladies sexuellement transmissibles, il est noté que "même si cette intervention ne prémunit pas des accidents pouvant survenir dans la vie sexuelle des jeunes filles accueillies (la réalité nous l'a d'ailleurs prouvé à plusieurs reprises cette année), elle permet de les responsabiliser et de leur faire prendre conscience de certains dangers". Considérées comme vulnérables aux risques sexuels, les jeunes sont la cible privilégiée des actions de prévention et de sensibilisation ${ }^{(27)}$, et encore plus lorsqu'il s'agit de jeunes femmes ${ }^{(28)}$. Mais lorsque en plus d'être jeunes et femmes, elles sont racialisées, le discours concernant leur sexualité n'échappe pas à des analyses normatives sur l'appétit sexuel, le nombre de partenaires ou sur la nature des relations sexuelles et affectives.

Au cours de l'année 2008, il y a eu de longues discussions au foyer à propos d'une jeune femme qui s'est vu offrir des chaussures et une robe par un homme plus âgé. Cette situation que la jeune nomme "avoir un pigeon" a créé un grand malaise dans l'équipe, bien que ce ne soit pas la première fois que des soupçons de prostitution apparaissent au foyer. Le fait que la jeune parle librement, et avec joie, de ces cadeaux pose un dilemme moral à l'équipe, comme le soulignent les propos d'une éducatrice : "Elle dit qu'elle le connaît depuis plusieurs semaines, qu'il a l'âge de son grand-père, qu'il est marié avec plusieurs femmes. La jeune dit qu'elle ne couche pas avec mais c'est impossible, il y a certainement une relation sexuelle derrière, sinon pourquoi lui offre-t-il des cadeaux ${ }^{(29)}$ ?" Les discussions en équipe portaient alors non pas sur la prostitution en général mais sur "le système africain du pigeon". Il ne s'agit pas uniquement d'un déplacement sémantique mais d'une autre matrice d'analyse et d'un nouveau cadre d'injonctions. Marie, en effet, a été identifiée comme responsable de ce qui lui arrivait. Pour certains, cette situation était due à son goût pour les vêtements. Pour d'autres, la raison résidait dans sa grande naïveté ou son besoin d'affection dû à son isolement familial. Le discours a ensuite changé pour évoquer avant tout l'origine africaine de Marie, faisant de cette sexualité transactionnelle ${ }^{(30)}$ une question d'ordre ethnique. Si l'on suit ce discours, Marie ne peut s'en sortir seule car elle est sous le contrôle de forces qui la dépassent, comme l'a fait remarquer le chef de service au cours d'une réunion : "Dans ce cas précis, on ne peut pas être 24 heures sur 24 avec elle, ce qu'il faudrait c'est la protéger d'elle-même $e^{(31)}$." Le foyer a reposé la problématique selon un angle où les raisons sont forcément les origines ethniques et non pas des pressions familiales et/ou économiques, ni des réseaux de sociabilité défaillants/déviants, comme c'est le cas pour la prostitution. 


\section{Un pigeon qui fait son nid}

Dans ce nouveau cadre d'analyse, les affirmations des éducateurs portent maintenant sur la différenciation entre la France et la Guinée, pays d'origine de Marie : "Tu n'es pas obligée de faire ça, en France les femmes travaillent et sont indépendantes ${ }^{(32) ", ~ l u i ~ d i s a i t ~}$ une éducatrice lors d'une discussion dans la cuisine. Il apparaît clairement que l'institution tente de mettre de la distance entre la jeune femme et ce qui est identifié comme sa culture, à l'instar de ce que décrit Nacira Guénif-Souilamas : "La première [injonction] portée par les acteurs institutionnels exhorte les filles à s'émanciper de la culture totalisante et oppressive de leur famille. Cette injonction accrédite une vision européocentrée des cultures allogènes, dont les accents dépréciateurs ont une troublante ressemblance avec les visées civilisatrices de la colonisation ${ }^{(33)}$." L'institution n'a pas cherché à contextualiser cette pratique du "pigeon" et à en comprendre les enjeux sociaux mais a vite trouvé dans les stéréotypes de la culture d'origine les raisons suffisantes pour l'expliquer. Ainsi, au nom d'une différenciation entre des pratiques culturelles anciennes et des pratiques modernes, Marie doit se conformer à un nouveau modèle, en adéquation avec sa nouvelle vie en France.

Quelques mois après "l'histoire du pigeon", au cours d'une discussion avec Marie, elle m'explique qu'elle ne sort plus qu'avec des "Blancs" parce qu'ils sont complètement différents des "Renois" qu'elle a connus par le passé : "Ils [les Blancs] sont plus doux et moins jaloux mais ils ne donnent pas beaucoup de cadeaux ${ }^{(34)}$."

Bien que l'équipe n'ait jamais dit qu'il valait mieux avoir des relations avec un groupe au détriment d'un autre, nous ne pouvons pas ignorer l'existence d'un "message secondaire" qui encourage la jeune femme à s'émanciper de la culture d'origine et à adhérer aux normes de la culture dominante. Comme le note Christelle Hamel : "Dans un contexte où la figure de 'l'homme arabe' est celle d'un homme machiste, l'appel à s'émanciper contient un message secondaire : il invite les filles à construire leur vie affective et sexuelle avec un homme du groupe majoritaire, censé être plus moderne et moins sexiste ${ }^{(35)}$."

\section{Genre et ethnie : les ressorts d'une double discrimination}

En plus des discussions individuelles avec Marie, l'équipe a cherché à intervenir sur l'ensemble de la population accueillie en mettant en place un partenariat avec l'Amicale du nid. Cette association qui lutte pour l'abolition de la prostitution est intervenue dans le foyer selon deux modalités : des séances de groupe auxquelles les éducateurs du foyer ne sont pas conviés et des séances dans les locaux de l'association auxquelles 
la jeune fille peut être accompagnée par un éducateur si elle le souhaite. En somme, la réponse proposée par le foyer face au "pigeon" est passée d'une approche spécifique liée à Marie en tant que femme et puis en tant que Guinéenne à une approche systémique où toutes les jeunes femmes sont potentiellement susceptibles d'être tentées par la prostitution. On peut à ce stade faire l'hypothèse que, face à cette augmentation de la population africaine dont fait mention le dernier rapport d'activité et donc d'un risque supposé plus important de pratiques déviantes, la réponse est de généraliser l'intervention de l'Amicale du nid. Ainsi, la racialisation de la population accueillie augmente le risque de "pigeonnage", ce qui nécessite un suivi systématique de toutes les jeunes femmes prises en charge.

Cette approche contient en fait une double ségrégation de genre. D'abord, parce que le fait qu'il s'agisse de jeunes femmes explique la position volontariste et préventive exprimée par les éducateurs dans les mois qui ont suivi cette affaire. Ensuite, parce que l'origine ethnicisée de ce "pigeon" explique le rapprochement avec l'Amicale du nid et un traitement systémique des jeunes femmes accueillies. Ainsi les séances collectives sont obligatoires et un compte rendu des présences est fait pour l'Aide sociale à l'enfance. Les raisons qui ont amené cette association à intervenir au foyer ont été clairement identifiées par les jeunes femmes d'origine africaine comme une réponse à l'histoire du "pigeon", et elles ont été jusqu'à inventer une expression pour exprimer leur point de vue : "le pigeon chie sur le nid".

L'intersection des rapports de genre et de "race" dans la pratique éducative ne s'arrête pas à des situations extrêmes comme les soupçons de prostitution ou de violences, mais elle est diffuse dans le quotidien, comme le montrent certaines discussions informelles sur la sexualité des jeunes femmes racialisées : "Celle-là, si elle n'a pas tiré son coup, elle ne sera pas calme $e^{(36) ", ~ " B . ~ d i t ~ q u ' e l l e ~ a ~ b e s o i n ~ d ' a v o i r ~ p l u s i e u r s ~ m e c s, ~ s i n o n ~ e l l e ~}$

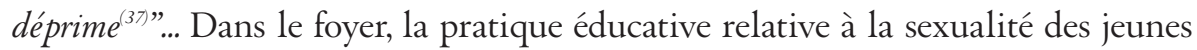
femmes s'exerce à partir d'un double prisme. Premièrement, c'est à travers les jugements moraux sur la sexualité des femmes que l'institution et les éducateurs réagissent, comme on l'a vu avec Marie, considérée au départ comme responsable de ce qui lui arrivait. Deuxièmement, c'est avec une approche différenciée entre racialisées et non-racialisées que le foyer ajuste son discours et ses actions.

\section{Conclusion}

Cette réflexion n'épuise ni les différentes facettes d'implication des rapports de genre et de "race" dans l'accompagnement éducatif au foyer, ni l'analyse des représentations des jeunes femmes et des éducateurs dans leur quotidien institutionnel. Nous avons 
choisi d'examiner comment le travail social dans un foyer pour jeunes femmes placées est traversé par deux rapports de pouvoir spécifiques. En effet, le travail social dans une institution semi-ouverte, comme le foyer étudié, est un travail de proximité où des liens forts unissent les jeunes femmes à l'équipe éducative. Cette relation n'est pas figée, elle évolue en fonction du temps passé par ces jeunes femmes dans l'institution. Dans ce cadre, le sexe de l'éducateur et son origine peuvent à la fois être des ressources qui influencent la nature de la relation, des obstacles qui la figent dans un cadre minimum d'échange, des rapports de pouvoir entre les acteurs ou encore des outils qui permettent d'analyser et de comprendre les enjeux de cette relation de proximité. En somme, considérer les rapports sociaux dans le travail social à travers le prisme du genre et de la "race" favorise la description des pratiques professionnelles et permet de questionner le travail éducatif. Cette approche permet aussi de conclure que le travail social est en proie à des difficultés spécifiques, surtout lorsqu'on voit qu'au bout de quatre ans de prise en charge par l'Aide sociale à l'enfance et six ans de vie en France, Marie se trouve obligée de quitter le territoire et de retourner en Guinée parce qu'elle ne remplit pas les conditions d'obtention d'un titre de séjour. Comment alors penser le lien entre genre, "race" et travail de proximité lorsque le statut des jeunes femmes comme celui des salariés est en proie à de plus en plus de précarité ?

\section{Notes}

1. JeannineVerdès-Leroux, Le Travail social, Paris, Éditions de Minuit, 1978.

2. Jean-Marie Domenach, Philippe Meyer, Paul Thibaud, "Pourquoi le travail social ?", in Esprit, avril-mai, 1972.

3. Jacques Ion, Jean-Pierre Tricart, Les Travailleurs sociaux, Paris, La Découverte, 1998.

4. Jacques T. Godbout, Michel Chauvrière (dir.), Les Usagers entre marché et citoyenneté, Paris, L'Harmattan, 1992.

5. Anselm Strauss, La Trame de la négociation. Sociologie qualitative et interactionnisme, Paris, l'Harmattan, 1992.

6. Castel Robert, Les Métamorphoses de la question sociale, Paris, Gallimard, 1995.

7. L’apport du concept de genre dans l'analyse des rapports sociaux est multiple. Voir Laure Bereni, Sébastien Chauvin, Chauvin, Alexandre Jaunait, Anne Revillard, Introduction aux gender studies, manuel des études sur le genre, Bruxelles, De Boeck, 2008, pp. 5-6.

8. Le terme de "race" est mis entre guillemets car ce n'est pas d'essence biologisante dont il est question mais de relations raciales, processus par lequel les individus sont hiérarchisés entre groupes majoritaires et minoritaires ("Blancs"/"non-Blancs") à partir d'un discours naturalisant basé sur des traits physiques et des différences phénotypiques. Lorsque les stéréotypes mobilisés ne s'arrêtent pas à des traits physiques et utilisent d'autres particularités, notamment culturelles (langue, religion, coutumes...), on parle d'“ethnicité". Voir Françoise Lorcerie (dir.), L'École et le Défi ethnique. Éducation et intégration, Paris, INRP, 2003, p. 38. 
9. Marc Bessin, "Les hommes dans le travail social : le déni du genre", in Yvonne Guichard-Claudic, Danièle Kergoat, Alain Vilbrod, L'Inversion du genre : Quand les métiers masculins se conjuguent au féminin... et réciproquement, Rennes, Presses universitaires de Rennes, 2008.

10. Corinne Saint-Martin, Etre assistante de service social. Effets de position et dynamique des pratiques en organisation, Paris, l'Harmattan, 1999.

11. Empan, $\mathrm{n}^{\circ}$ 61, "Des femmes et des hommes un enjeux pour le social", 2007 ; Travailler le social, $\mathrm{n}^{\circ} 41$, "Genre et travail social", $2008 ;$; VST, n¹06, "Que faire avec les filles", 2010.

12. Valérie Amiraux, Patrick Simon, "There Are No Minorities Here: Cultures of Scholarship and Public debate on immigrants and integration in France", in International Journal of Comparative Sociology, vol. 47, n 3-4, 2006.

13. Abdelmalek Sayad, "Qu'est-ce qu'un immigré ?", in Peuples méditerranéens, vol. 7,1979.

Sossie Andezian, Jocelyne Streiff-Fenart, "Réseaux féminins dans l'immigration maghrébine : régulations pratiques et rôle symbolique", Centre de recherches et d'études sur les sociétés méditerranéennes (CRESM), vol. 20, Paris,

éditions du CNRS, 1982. http://aan.mmsh.univ-aix.fr/volumes/1981/Documents/Etudes_Reseaux-feminins.pdf

14. Hanna Malewska-Peyre, Crise d'identité et déviance chez les jeunes immigrés, Vaucresson, CFRES, 1982 ;

Faïza Guelamine, Intervenir auprès des populations immigrées, Paris, Dunod, 2000.

15. Fatima Ait Ben Lmadani, "Dynamiques du mépris et tactiques des 'faibles", in Sociétés contemporaines, $\mathrm{n}^{\circ} 70,2008$.

16. Christine Delphy, "Libération des femmes, année zéro", in Partisans, $n^{\circ} 54 / 55,1970$.

17. Christine Delphy, L'Ennemi principal. Économie politique du patriarcat, Paris, Syllepse, 1998.

18. Réunion d'équipe du mardi, février 2008.

19. Anne-MarieArborio, Un personnel invisible. Les aides soignantes à l'hôpital, Paris, Anthropos, 2001.

20. Jacqueline Laufer, Chaterine Marry, Margaret Maruani(dir.), Le Genre au travail. Les sciences sociales à l'épreuve des différences de sexe, Paris, La Découverte/MAGE, 2003.

21. Michel Wieviorka, "La production institutionnelle du racisme", in Hommes \& Migrations, $n^{\circ} 1211,1998$.

22. Rapport d'activité 2009.

23. Anne Loyer, "Représentations et pratiques des travailleurs sociaux face à l'interculturalité", in Emmanuel Jovelin (dir.), Le Travail social face à l'interculturalité, comprendre la différence dans les pratiques d'accompagnement social, Paris, L'Harmattan, 2002, p. 164.

24. Rapport sur le transfert, document interne lié à l'évaluation d'un voyage réalisé avec les jeunes femmes courant 2009.

25. Colette Guillaumin, Sexe, race et pratique du pouvoir. Lidée de nature, Paris, Côté Femmes, 1992 ; Christelle Hamel, "La sexualité entre sexisme et racisme : les descendant(e)s de migrant(e)s originaires du Maghreb et la virginité", in Nathalie Benelli, Christine Delphy, Jules Falquet, Christelle Hamel, Ellen Hertz, Patricia Roux, Nouvelles Questions Féministes, $\mathrm{n}^{\circ}$ 25, "Sexisme et racisme : le cas français", 2006.

26. Elsa Dorlin (dir.), Sexe, classe, race, pour une épistémologie de la domination, Paris, PUF, 2009 ; Christian Poiret, "Articuler les rapports de sexe, de classe et interethniques : quelques enseignements du débat nord-américain", in Revue européenne des migrations internationales, vol. 21, $\mathrm{n}^{\circ} 1,2005$.

27. Michel Bozon, Sociologie de la sexualité, Paris, Nathan, 2002.

28. Nathalie Bajos, Michel Bozon (dir.), Enquête sur la sexualité en France. Pratiques, genre et santé, Paris, La Découverte, 2009.

29. Réunion d'équipe du mardi, mai 2008.

30. Michelle Poulin, "Sex, money and premarital partnerships in southern Malawi", in Social Science and Medicine, 2007.

31. Réunion d'équipe du mardi, septembre 2008.

32. Novembre 2008 dans la cuisine du foyer.

33. Nacira Guenif Souilamas, Des "beurettes" aux descendantes d'immigrants nord-africains, Paris, Grasset, 2000, p. 25.

34. Discussion avec Marie, mai 2009.

35. Christelle Hamel, art. cit., p. 46.

36. Pendant la réunion du mardi, février 2009. Une éducatrice me disait cela en commentaire d'une discussion sur le suivi d'une jeune femme et sous forme de plaisanterie.

37. Un éducateur parlant de la situation d'une jeune femme dont il est le référent, novembre 2009, pendant la réunion d'équipe du mardi. 